Utah State University

DigitalCommons@USU

$5-12-2020$

\title{
Random Encounter and Staying Time Model Testing with Human Volunteers
}

\author{
Laura Garland \\ University of Alberta \\ Eric Neilson \\ Canadian Forest Service Natural Resources Canada \\ Tal Avgar \\ Utah State University \\ Erin Bayne \\ University of Alberta \\ Stan Boutin \\ University of Alberta
}

Follow this and additional works at: https://digitalcommons.usu.edu/eco_pubs

Part of the Ecology and Evolutionary Biology Commons

\section{Recommended Citation}

Garland, L., Neilson, E., Avgar, T., Bayne, E. and Boutin, S. (2020), Random Encounter and Staying Time Model Testing with Human Volunteers. Jour. Wild. Mgmt.. doi:10.1002/jwmg.21879

This Article is brought to you for free and open access by the Ecology Center at DigitalCommons@USU. It has been accepted for inclusion in Ecology Center Publications by an authorized administrator of DigitalCommons@USU.

For more information, please contact

digitalcommons@usu.edu.

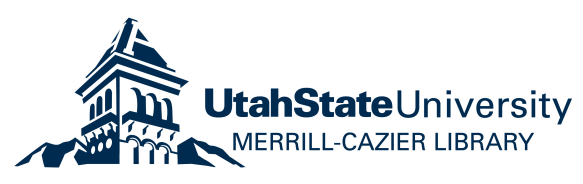




$$
29 \text { April } 2020
$$

Laura Garland

University of Alberta

116 Street and 85 Avenue

Edmonton, AB T6G 2R3

780-492-3111

lgarland@ualberta.ca

RH: Garland et al. • Density Estimation

9 Random Encounter and Staying Time Model Testing with Human Volunteers

LAURA GARLAND ${ }^{1}$ University of Alberta, 116 Street and 85 Avenue, Edmonton, AB T6G 2R3, Canada

ERIC NEILSON, Canadian Forest Service Natural Resources Canada, 5320-122 Street, Edmonton, AB T6H 3S5, Canada

TAL AVGAR, Utah State University, Old Main Hill, Logan, UT 84322, USA

ERIN BAYNE, University of Alberta, 116 Street and 85 Avenue, Edmonton, AB T6G 2R3, Canada Canada

ABSTRACT Ecology and management programs designed to track population trends over time increasingly are using passive monitoring methods to estimate terrestrial mammal densities.

21 Researchers use motion-sensing cameras in mammal studies because they are cost-effective and

22 advances in statistical methods incorporate motion-sensing camera data to estimate mammal

23 densities. Density estimation involving unmarked individuals, however, remains challenging and

24 empirical tests of statistical models are relatively rare. We tested the random encounter and

25 staying time model (REST), a new means of estimating the density of an unmarked population,

${ }^{1}$ Email: lgarland@ualberta.ca 
Garland et al.

26 using human volunteers and simulated camera surveys. The REST method produced unbiased

27 estimates of density, regardless of changes in human abundance, movement rates, home range

28 sizes, or simulated camera effort. These advances in statistical methods when applied to motion-

29 sensing camera data provide innovative avenues of large-mammal monitoring that have the

30 potential to be applied to a broad spectrum of conservation and management studies, provided

31 assumptions for the REST method are rigorously tested and met.

32 KEY WORDS density, human volunteers, mammals, motion-sensing camera, random encounter

33 and staying time method, REST.

34 Abundance and density are fundamental ecological parameters that are difficult to measure

35 because individuals move in and out of sample plots, and not all individuals present at sample

36 units are detected (Royle and Nichols 2003). Heterogeneity in individual movement and

37 presence at sample units necessitates estimating and correcting for the probability of detection.

38 Count data from repeated surveys of sampling units fundamentally inform abundance estimates

39 corrected for detection. Capture-mark-recapture (CMR) uses the marked individual as the sample

40 unit with the pattern of captures over time assisting with abundance estimates (Seber 1982). In

41 these cases, the model allows heterogeneity of capture probability among individuals (Pollock

42 1982).

43 Ambiguity in the area over which researchers estimate abundance can make translating

44 abundance into density (i.e., number/unit area) less than straightforward. Individuals living on

45 the boundary of the study area substantially affect density estimates (Efford 2004). Spatially

46 explicit capture recapture (SECR) models use the spatial pattern in the recaptures of individuals

47 to estimate probable locations of home range centers within a study area to address this issue

48 (Efford 2004, Royle et al. 2013). 
Garland et al.

Chandler and Royle (2013) built on the SECR model to consider sampling site locations and their associated count statistics to estimate density without the need for marking individuals.

51 This model infers the number and locations of home range centers from the spatial

52 autocorrelation of the count data. Surveyors must space sampling sites so an individual can

53 encounter multiple traps, in contrast with the assumption of site independence assumed with 54 previous models.

Researchers now commonly use motion-sensitive cameras to estimate habitat use,

56 distribution, abundance, and density for unmarked wildlife populations (Burton et al. 2015).

57 Minimal human intervention, reduced cost, and simplified logistics make camera surveys

58 attractive for high profile species of conservation concern or in conditions that prevent direct

59 observation or capture of individuals. Photos that identify individuals are useful in standard

60 CMR methods, but photos of species that do not allow for individual identification can also be

61 used to calculate abundance estimates using SECR models (Royle and Nichols 2003, Royle

62 2004) and density (Chandler and Royle 2013, Ramsey et al. 2015).

By assuming individuals encounter point detectors randomly, Rowcliffe et al. (2008)

64 developed the random encounter model (REM). The REM uses independent estimates of travel

65 speed (obtained through observation), time active each day, group size, and the area of the

66 detection zone of each camera to relate photos/time to density (Rowcliffe et al. 2008). The model

67 assumes that samples from each camera are independent and uses count data (photos/unit time)

68 for estimations but bases estimation on individual movement rather than inferred spatial point

69 process. The model depends on accurately estimating movement speed, time active, and group

70 size, necessitating considerable additional effort that may not be possible for many species.

71 Rowcliffe et al. (2016) present suggestions on feasible approaches. 
Garland et al.

Nakashima et al. (2018) modified Rowcliffe’s original method to measure the staying time of an individual within the detection area of remote cameras. They referred to this model as the random encounter and staying time (REST) method. The REST model assumes that researchers place cameras randomly relative to individual movement within the study area. With this assumption, the residence time of an individual at any given detector is a function of the duration of time the detector is deployed and the proportion of the study area it samples. Under the assumption of random movement, residence time scales linearly with the number of individuals, thereby allowing an estimate of density without the need to estimate rate of movement, home range size, or individual identity. The model also does not require closure of the study area in the sense that individuals do not leave or enter the area, but only that immigration, emigration, births, and mortality are balanced during the study period. The REST model calculates population density as a function of the residency time the target species spends in front of a camera. The equation, modified from Nakashima et al. (2018) to account for potentially different sampling durations and areas between cameras, is:

$$
\hat{\rho}=\frac{\sum_{i=1}^{n} t_{i}}{\sum_{i=1}^{n} T_{i} \times \mathrm{a}_{i}},
$$

where $\hat{\rho}$ is the estimated density, $n$ is the number of cameras, $t_{i}$ is the staying time of an individual at the $i$ th camera, $T_{i}$ is the time the $i$ th camera was active, and $a_{i}$ is the area sampled by the ith camera (its 100\% detection zone; s in Nakashima et al. 2018).

If a camera records multiple individuals at the same time, the model estimates residency time independently for each individual. Importantly, calculating the cumulative residence time ( $t$ in the above equation) does not require identifying distinct residency bouts, eliminating the need 
Garland et al.

94 to define a camera detection, simply sum the time individuals spend in front of each camera. This

95 method is applicable to territorial and non-territorial species, provided researchers distribute cameras randomly in relation to animal space-use patterns (i.e., no baiting or placing cameras

97 only in areas with preferred habitat characteristics).

The REST model assumes that cameras sample habitat proportional to their availability.

99 The precision and accuracy of estimates to movement within the territory or home range relies 100 on the assumption of equal probability of a home range existing within the study area (i.e.,

101 homogeneity of the point-pattern describing the distribution of home ranges). Additionally, 102 detectability within the detection zone of the cameras must be perfect $(p=1)$. This method also 103 assumes the detection device does not modify individual movement.

104 Nakashima et al. (2018) tested the REST model using computer simulations and field 105 surveys of duiker populations (red forest duiker [Cephalophus natalensis] and blue duiker 106 [Philantomba monticola]) in Moukalaba-Doudou National Park, Gabon. The REST model 107 provided unbiased estimates of abundance for nearly all simulated populations representing 108 paired and solitary movement, continuous movement, and movement with resting. The REST 109 estimates from camera surveys of actual duiker populations were similar to estimates made via 110 line transect surveys. Nakashima et al. (2018) provided strong evidence for the robustness of the 111 REST method in computer simulations, but they did not know the true densities of the duiker 112 populations they tested.

We sought to test the REST method using known densities of human volunteers, which 114 provided us with proof of concept. Human volunteers were advantageous because they allowed 115 for more realistic movement paths than computer simulations. Our objective was to determine if 116 movement rate, home range size, and density affected bias and precision of the estimates 
Garland et al.

117 produced by REST. We equipped human volunteers with global positioning system (GPS)

118 devices and gave them precise movement rules such that home range size and movement rate

119 were varied.

120 STUDY AREA

121 Our test took place at the Louise McKinney Riverfront Park in Edmonton, Alberta, Canada

$122\left(53^{\circ} \mathrm{N} 113^{\circ} \mathrm{W}\right)$ on 16 and 23 September 2017. The entire park is approximately 4.0 ha in size and

123 the weather on both days was clear and sunny $\left(\sim 15^{\circ} \mathrm{C}\right)$. The study area was approximately 1.5

124 ha in size, and consisted of flat, open, grassy areas, walking paths, and a pavilion, all of which

125 were accessible to the volunteers.

\section{METHODS}

127 The Research Ethics Office at the University of Alberta granted approval for using human

128 volunteers in our test (application number pro00075181). We employed 12 volunteers as proxies

129 for non-territorial, unmarked terrestrial mammals. We assigned volunteers to use first the entire

130 park and then half the park as their home range. We designated home range boundaries with

131 flags. We gave each volunteer either a GPSMap64 or a GPSMap78 unit (Garmin, Olathe, KS,

132 USA), both of which are accurate within 5-10 m to track their movements every second for the

133 duration of each scenario.

134 We conducted 6 scenarios, each scenario being a different combination of movement

135 rates and home range sizes. Each scenario lasted 16 minutes and included 3 movement patterns

136 (jogging for 10 minutes and resting for 6 minutes, walking for 10 minutes and resting for 6

137 minutes, and walking for 16 minutes continuously) performed within 2 home range sizes (0.75

138 ha and $1.50 \mathrm{ha}$ ). We instructed volunteers to move independently of each other during each test

139 but we synchronized their movement and rest periods. 
Garland et al.

We tracked the duration of each scenario using a stopwatch and used a whistle to signal when subjects were to change movement rates and end each scenario. Because of variation among volunteers in the time they took to start, stop, and save their individual tracks, each scenario varied slightly from 960 seconds (16 min; Table 1). We merged tracks collected over both days according to scenario in ArcMap version 10.5.1 (Esri, Redlands, CA, USA) and clipped each track to the shortest duration of any given volunteer within scenarios to standardize the number of points per person per scenario (932 \pm 19 [SD] seconds). We created polygons consisting of 800 cells around each scenario based on the coordinates of the outermost tracks (Fig. 1). Each cell was approximately $20 \mathrm{~m}^{2}$. We summed the number of points per cell for each scenario as a proxy of time spent in each cell. If a point fell on the border of 2 adjacent cells, we randomly assigned it to 1 cell.

We assumed the habitat characteristics in the study area were homogenous during this study, and detectability was perfect given that GPS units tracked each volunteer and never failed during the simulations. Volunteers were not attracted to detection devices because we did not actually deploy any cameras.

We varied human densities to include 2, 6, and 12 people. We varied sampling effort by varying the number of cells selected randomly as camera deployments. We selected $8,20,50$, or 100 cells as camera deployment sites, resulting in 1\%, 2.5\%, 6.25\%, and $12.5 \%$ coverage of the study area, respectively. We used 1,000 bootstrap samples with replacement of camera effort in each scenario of movement speed, human densities, and home range area for 72 different scenarios in R (R version 3.5.1, www.r-project.org, accessed 10 Oct 2018). We estimated the density of human volunteers across each combination of movement speed, true human density, and home range area using equation 1 . We then multiplied the resulting density by the area to 
Garland et al.

163 calculate abundance for comparison to the number of volunteers per scenario. We calculated 164 means and confidence intervals across bootstrapped samples to estimate abundance and quantify 165 precision (data and r code available online in Supporting Information).

\section{RESULTS}

167 The REST model provided accurate estimates of human density regardless of movement rate, 168 home range area, camera effort, or number of volunteers (Fig. 2). Precision decreased when our 169 sampling effort was low (i.e., 1\% coverage). Neither movement rate nor home range size 170 affected estimator accuracy, although the REST model consistently estimated abundance with 171 lower precision under walking-and-resting and jogging-and-resting scenarios compared to 172 scenarios representing homogenous walking speeds.

In scenarios representing human densities of 2 people, we observed the least amount of

174 error across all movement or home range size. In scenarios with 20 and 50 cameras, as human

175 abundance in the park increased, precision decreased.

Across all home range sizes and movement rates, the REST method accurately estimated

177 human densities. We found no effect of home range size on estimator accuracy or precision.

178 Estimators provided the greatest precision under continuous-walking scenarios across all levels 179 of camera effort, human density, and home range size. The introduction of heterogeneity in 180 movement rate did not affect estimator accuracy but did reduce precision.

Not surprisingly, estimator precision increased with camera effort. With 100 camera

182 cells, confidence intervals were, on average, an order of magnitude smaller than in scenarios 183 with 8 camera cells (Fig. 2).

184 DISCUSSION 
Garland et al.

185 The REST method accurately estimated human densities regardless of movement rate, home

186

187

188

189

190

191

192

193

194

195

196

197

198

199

200

201

202

203

204

205

206

207

range size, and camera coverage in these scenarios. Increased density resulted in decreased

precision because of the increased variability of staying times across cameras. Although

movement rate and home range size did not affect estimator error, estimators were least precise

in scenarios involving resting. Increased precision when volunteers were moving at slower paces

continuously as opposed to moving and resting supports the theory that homogenous movements rates result in more precise estimates. Nakashima et al. (2018) noted that the REST method may

be less precise for species that have long periods of inactivity because cameras rarely capture the target animal resting. Our human scenarios partially accounted for this potential bias by

incorporating resting, in which volunteers did not move from their locations for approximately $38 \%$ of the survey period during 2 of the scenarios. Despite this lack of movement, the REST method was still able to estimate density in those scenarios; however, the estimates were less precise than other movement rates. Further testing of the effects of species with long periods of inactivity may be warranted. We deviated from Nakashima et al. (2018) by using boot-strapping rather than likelihood-based quantification of uncertainty. As such, we demonstrate the potential for unbiased estimation of staying time even where it does not necessarily follow a parametric distribution.

Nakashima et al. (2018) also suggested that cameras have sensitive sensor settings, no delay period between photos, or alternatively, take video recordings, and that the effective detection area be tested in situ according to methods proposed by Rowcliffe et al. (2011). We excluded potential effects of delayed camera capture rates and imperfect detectability by having each volunteer tracked every second. Camera capture rates and imperfect detectability, however, could present challenges in field settings when researchers use real cameras. 
Garland et al.

Environmental variation or attributes of the study species may influence detectability.

209

210

211

212

213

214

215

216

217

218

219

220

221

222

223

224

225

226

227 increase the precision of estimates. This method offers a cost-effective, unbiased means to

228 estimate animal densities from motion-sensitive camera data without the use of marked

229 individuals or estimates of home range sizes. The application of the REST method to motion- 
Garland et al.

230 sensing camera studies may have the potential to improve monitoring efforts for several species, 231 provided assumptions are met.

\section{ACKNOWLEDGMENTS}

233 We thank the University of Alberta Research Ethics Office for allowing us to complete this

234 study, in addition to the 12 volunteers who participated in the park scenarios. We also thank E.

235 K. for helpful comments and feedback on an earlier draft of this manuscript. Finally, we thank

236 the University of Alberta and Natural Sciences and Engineering Research Council of Canada

237 (NSERC) CREATE-EI scholarships for their financial support during this study.

238

239

240

241

242

243

244

245

246

247

248

249

250

251

252

\section{LITERATURE CITED}

Anile, S., and S. Devillard. 2016. Study design and body mass influence RAIs from camera trap studies: evidence from the Felidae. Animal Conservation 19:35-45.

Burton, C. A., E. Neilson, D. Moreira, A. Ladle, R. Steenweg, J. T. Fisher, E. Bayne, and S. Boutin. 2015. Wildlife camera trapping: a review and recommendations for linking surveys to ecological processes. Journal of Applied Ecology 52:675-685.

Chandler, R. B., and J. A. Royle. 2013. Spatially explicit models for inference about density in unmarked or partially marked populations. Annals of Applied Statistics 7:936-954.

Efford, M. 2004. Density estimation in live-trapping studies. Oikos 106:433-636.

Nakashima, Y., K. Fukasawa, and H. Samejima. 2018. Estimating animal density without individual recognition using information derivable exclusively from camera traps. Journal of Applied Ecology 55:735-744.

Pollock, K. H. 1982. A capture-recapture design robust to unequal probability of capture. Journal of Wildlife Management 46:757-760. 
Garland et al.

253 Ramsey, D. S. L., P. A. Caley, and A. Robley. 2015. Estimating population density from presence-absence data using a spatially explicit model. Journal of Wildlife Management

Rovero, F., F. Zimmermann, D. Berzi, and P. Meek. 2013. "Which camera trap type and how many do I need?” A review of camera features and study designs for a range of wildlife research applications. Hystrix 24:148-156.

Rowcliffe, J. M., C. Carbone, P. A. Jansen, R. Kays, and B. Kranstauber. 2011. Quantifying the sensitivity of camera traps: an adapted distance sampling approach. Methods in Ecology and Evolution 2:464-476.

Rowcliffe, J. M., J. Field, S. T. Turvey, and C. Carbone. 2008. Estimating animal density using camera traps without the need for individual recognition. Journal of Applied Ecology 45:1228-1236.

Rowcliffe, J. M., P. A. Jansen, R. Kays, R. Kranstauber, and C. Carbone. 2016. Wildlife speed cameras: measuring animal travel speed and day range using camera traps. Remote Sensing in Ecology and Conservation 2:73-118.

Royle, J. A. 2004. N-mixture models for estimating population size from spatially replicated counts. Biometrics 60:108-115.

Royle, J. A., R. B. Chandler, C. C. Sun, A. K. Fuller. 2013. Integrating resource selection information with spatial capture-recapture. Methods in Ecology and Evolution 4:501-600.

Royle, J. A., and J. D. Nichols. 2003. Estimating abundance from repeated presence-absence data or point counts. Ecology 84:777-790.

Seber, G. A. F. 1982. The estimation of animal abundance and related parameters. Blackburn Press, Macmillan, New York, USA. 
Garland et al.

276 Tobler, M. W., S. E. Carrillo-Percastegui, R. Leite Pitman, R. Mares, and G. Powell. 2008. An

277 evaluation of camera traps for inventorying large- and medium-sized terrestrial rainforest

278 mammals. Animal Conservation 11:169-178.

279 Villette, P., C. J. Krebs, T. S. Jung, and R. Boonstra. 2016. Can camera trapping provide accurate

280 estimates of small mammal (Myodes rutilus and Peromyscus maniculatus) density in the

281 boreal forest? Journal of Mammalogy 97:32-40.

282 Associate Editor: Quresh Latif.

283 
Garland et al.

\section{Figure Captions and Tables}

285 Figure 1. Merged tracks of 12 human volunteers in the 800-cell polygon from scenario 5. In

286 scenario 5, the entire Louise McKinney Riverfront Park, Edmonton, Alberta, Canada, was

287 available to everyone on 16 and 23 September 2017, and the movement rate was walking for 10

288 minutes and resting (no movement) for 6 minutes.

289 Figure 2. Bootstrapped mean estimates and 95\% confidence intervals of human densities

290 including 2, 6, and 12 people with motion-sensitive camera effort of 8, 20, 50, and 100 cameras

291 across all 6 scenarios of movement rate and home range size in the Louise McKinney Riverfront

292 Park, Edmonton, Alberta, Canada on 16 and 23 September 2017. Small (purple) and large

293 (orange) in the legend refer to the home range size available to the volunteers, either 0.75 ha or

2941.5 ha, respectively. JogRest, Walk, and WalkRest refer to the movement rates in each scenario

295 and are differentiated by triangles, circles, and squares, respectively. 
Garland et al.

297 Table 1. Movement rates of the human volunteers and home range sizes (ha) available in each 298 scenario on 16 and 23 September 2017 at the Louise McKinney Riverfront Park, Edmonton, 299 Alberta, Canada, where the cell area $\left(\mathrm{m}^{2}\right)$ refers to the approximate cell size per scenario. We 300 recorded the duration of each scenario in seconds (s), and included the number of points tracked 301 per second (point freq).

302

\begin{tabular}{|c|c|c|c|c|c|c|}
\hline Scenario & $\begin{array}{l}\text { Home range } \\
\text { (ha) }\end{array}$ & Movement rate & $\begin{array}{l}\text { Duration } \\
\text { (s) }\end{array}$ & $\begin{array}{l}\text { Point freq } \\
\text { (s) }\end{array}$ & $\begin{array}{l}\text { Cell area } \\
\left(\mathrm{m}^{2}\right)\end{array}$ & $\begin{array}{l}\text { Total area } \\
\left(\mathrm{m}^{2}\right)\end{array}$ \\
\hline 1 & 0.75 & Jog 5 min, rest $3 \min (2 \times)$ & 11,424 & 952 & 20 & 16,000 \\
\hline 2 & 0.75 & Walk 5 min, rest $3 \min (2 \times)$ & 11,184 & 932 & 19 & 15,200 \\
\hline 3 & 0.75 & Walk continuously (16 min) & 11,268 & 939 & 20 & 16,000 \\
\hline 4 & 1.50 & Jog $5 \min$, rest $3 \min (2 \times)$ & 11,208 & 934 & 20 & 16,000 \\
\hline 5 & 1.50 & Walk 5 min, rest $3 \min (2 \times)$ & 10,752 & 896 & 20 & 16,000 \\
\hline 6 & 1.50 & Walk continuously (16 min) & 11,244 & 937 & 20 & 16,000 \\
\hline
\end{tabular}


Garland et al.

303 Summary for online Table of Contents

304 The random encounter and staying time (REST) method produced unbiased estimates of density, 305 regardless of home range size and movement rate of human volunteers. Although our tests 306 suggest the REST method may be a viable means of unmarked mammal density estimation, 307 further testing of the REST method may be warranted to account for species with varying body 308 sizes and periods of non-movement.

309

310

311 